\title{
Designing Group Annotations and Process Visualizations for Role-Based Collaboration
}

\author{
Gregorio Convertino, Anna Wu, Xiaolong (Luke) Zhang, Craig H. Ganoe, Blaine \\ Hoffman and John M. Carroll
}

\{gconvertino, auw133, lzhang, cganoe, bhoffman, jcarroll\}@ist.psu.edu

College of Information Sciences and Technology, The Pennsylvania State University, University Park, PA, 16802

\begin{abstract}
Team collaboration in situations like emergency management often involves sharing and management of knowledge among distributed domain experts. This study extends our previous research on improving common ground building among collaborators with role-based multiple views, and proposes prototypes of annotation and process visualization tools to further enhance common ground building. We illustrate specific needs through two problem scenarios and propose the designed prototypes through storyboarding.

"The costs of not doing enough in a coordinated way far outweigh the costs of doing it... in a coordinated, better way." "We are in a position to do so and we should do it". The EU commission environment spokesperson commenting on the recent forest fire tragedy in Greece, where at least 60 people died. When Greece requested help, nine EU countries responded within 48 hours and all the relief efforts were coordinated through a centre in Belgium (BBC News, Brussels, August 27 2007).
\end{abstract}

\section{Research Focus}

The quote above points to the criticality of coordination in emergency management.

Crisis management teams are an example of multi-expert teams making complex decisions in constrained conditions (e.g., limited time) and under uncertainty (i.e., based on incomplete or contradictory evidence). Analogous teams can be found in other work domains [e.g., 18].

Due to the complexity of the tasks, these teams usually consist of members who are specialized experts with diverse and stable roles. They contribute different perspectives and different areas of expertise useful to complete the task successfully. Different perspectives imply different goals, background knowledge, and assumptions, while different areas of expertise imply different languages and responsibilities [13]. Specialist knowledge is meaningful and useful in a specific application context. Trained multi-expert teams handle such mapping between knowledge and context implicitly. 
The goal for these teams is to make optimal decisions through timely and accurate sharing and coordination of expert knowledge. The work is made difficult by the intrinsic complexity of the task (e.g., massive amount of data, dynamic information, limited time, uncertainty), cognitive limitations of experts (e.g., limited capacity and judgment bias), and constraints that the tools (e.g., mixture of mobile and desktop, groupware) and the collaborative setting (e.g., distributed across places and working environments, limited time) impose.

We study ways in which collaborative technology can support decision-making by helping experts to handle the task complexity and by reducing their cognitive limitations or biases. This requires systematic analysis and modeling of behavior.

Over the last three years in our research program on common ground (CG), we have investigated the formation of group structures that regulate knowledge sharing and affect group performance. Specifically, we study the work of small teams of experts performing emergency management planning tasks on maps in distributed and synchronous settings. Building on observations of real practices in the field, we developed a reference task and a laboratory procedure for studying these types of teams [e.g. 6;8]. We used this experimental model as a test bed for studying regularities in the team process. Our findings are now informing the scenario-based design of tools for knowledge sharing, awareness, and decision-making.

This paper focuses on the design of tools that support knowledge sharing and decision-making in multi-role teams. We present the initial software prototype, which was adopted in our empirical study on emergency management teams [8]. Based on prior research and empirical observations, we isolate key needs of the teams, which we describe through scenarios. On this basis, we develop visualization and annotation tools.

\section{Prior Geocollaborative Prototype}

Figure 1 displays the user interface of the prototype (also described in [9]). In this paper, we augment this initial prototype with additional tools. The initial prototype features a team map and multiple role-specific maps (Figure 1). Each map displays multiple layers of geographical data. The team map (on the right) is a shared object that is used collaboratively by all the team members. The role-specific maps (on the left), different for each expert, contain unshared role-specific data layers as well as data layers also in the team map (shared data). A toolbar provides navigation and annotation functionality. However, in the experiments we found that the key limitation of this prototype is that, other than publishing information from role-specific map to team map and annotation list, the system does not provide support for information filtering or aggregation and activity monitoring and regulation. 


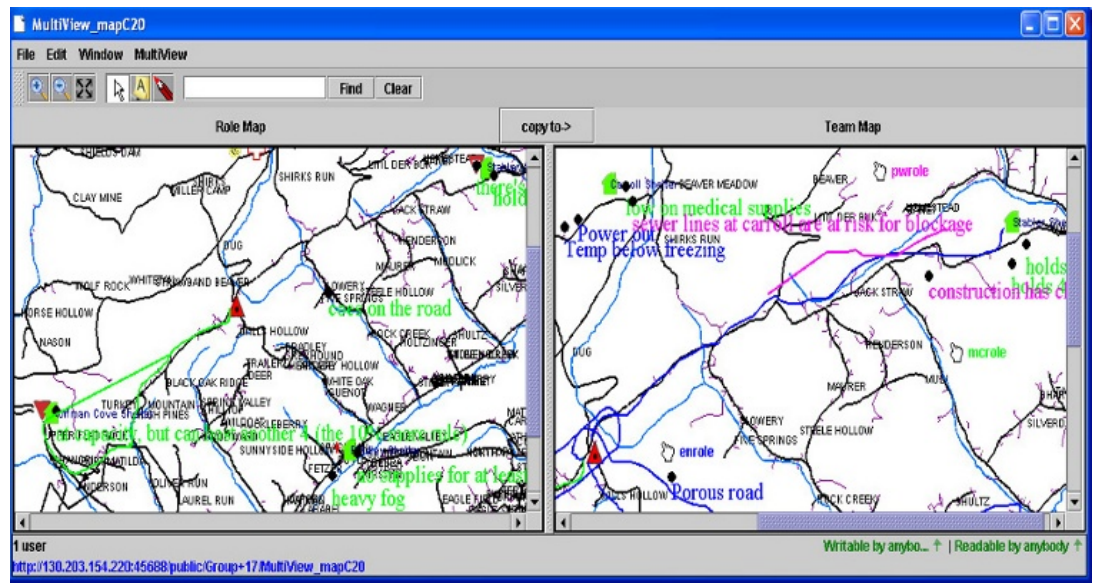

Figure 1: Geocollaborative prototype, showing role (left) and team (right) views along with annotation and telepointer features (also described in [9]).

\section{Annotation and Visualization Tools}

We propose the introduction of annotation and visualization tools in support of knowledge sharing and decision-making in multi-expert teams.

Prior research on annotations has shown that shared annotations address needs that are related but distinct from the needs addressed by personal annotations [19]. Group annotation tools (input or manipulation tools) enable externalizing judgments about information in context. The tagging of the annotations allows post-hoc categorization and bottom-up aggregation of facts or judgments. Annotations also allow for relating different pieces of information or integrating across different media. For example, an annotation in the geographic map can point to another annotation or to role-specific media such as external documents, charts, tables, photos, etc. Process-level annotations help the group manage the decision (e.g., how-to comments, queries on missing information (“?”), proposed categorizations, decisions in progress). These can be reused in similar situations: e.g., templates that can be reapplied in similar tasks.

Group process visualization tools (output or monitoring tools) enable presenting complex information in ways that can be easily perceived during decision-making (e.g., minimalist map overview, color-coding of roles), give a concrete form to abstract decision-making processes by showing records of actions in relation to geospace, time, roles, or decision alternative, and reduce the cognitive load (e.g., interactive animations can "link" information pieces spatially dispersed or role-specific). Process visualizations can provide the team with multiple alternative views of the de- 
cision process. We are exploring three design ideas: (1) A geo-spatial overview of the contributions that filters out details and displays what information was contributed 'where' on the map and 'by what expert' (e.g., a similar design concept is used for example in the Google Maps Mashups generated for Hurricane Katrina). (2) A temporal overview of the process: a n-timeline view (one timeline for each of the ' $n$ ' experts or roles) displaying 'who' did what 'type of operation' (add, change, delete), 'when', and 'temporal patterns' among the contributions, time spent in each task phase, and deadlines (e.g., see visualization tools in Ganoe et al. [11] and Andre' et al. [1]); (3) A conceptual view of the decision space: elements of risks and judgments contributed by each expert are grouped by the decision alternatives (or by content categories, or tags, defined by the team). The resulting breakdown of judgments is displayed either in a bar chart with alternative solutions (or content categories) defining the number of bars or a tabular representation with solutions as columns and pro or against judgments as rows (see the CACHE system in Billman et al. [4]). This gives the team a summative balance of the pro or against judgments shared.

Note that it is not our intent to develop new visualization techniques for particular data types. A large amount of research has been done in information visualization to visualize various types of data (e.g., list, 2D data, 3D data, temporal data, etc.). Often visualization designs focus on data type, task type, and individual cognition, but overlook information added during work (e.g., annotations) and group processes, which modulate how the initial data are perceived and how the tasks are performed. We believe that it is not sufficient to see visualization tools just as modules only dependent on data type and task type. It is important to investigate and model the relationship among data types, task type, and group process with and without the mediation of collaborative visualization tools.

\section{Identifying Needs based on Previous Research}

Evidence from research on group decision-making. Proposition 1: When using large and dynamic information corpora to make decisions under uncertainty, unsupported individuals and groups are both limited and biased.

We isolate three main systematic limitations or biases that affect groups' ability to accurately share knowledge and make decisions:

1. Groups' biased analysis and discussion. When sharing and discussing both shared and unshared information, collaborators tend to privilege familiar to unfamiliar information: unless corrective interventions are introduced, group discussions are systematically biased toward shared information to the expenses of unshared and less familiar information (see hidden profile in [17]). 
2. The limited capacity in processing massive information in a short interval of time and limited accuracy in distinguishing and managing distinct epistemological categories of information at the same time: facts and sources, inferences about facts and sources, how-to knowledge about the task, experts' related experience (e.g., [12]).

3. Groups' biased synthesis and decisions. When interpreting the meaning and weighting the relevance of information under uncertainty and social influence, group decisions are affected by systematic biases such as anchoring to early hypotheses, confirmation bias, and bias in posterior-probability estimates (e.g., [4]).

Evidence from research on collaborative technology. Proposition 2: Groups have self-regulation abilities that remain unexploited in face-to-face conditions but that could be exploited through appropriate collaborative technologies.

Since the early 1980s, various Group Decision-Making Support Systems (GDSS) have been designed with the aim of supporting groups in tasks such as brainstorming, collaborative editing, and meeting recording. Later, the emergence of a new generation of groupware or systems for Computer-Supported Cooperative Work (CSCW) has enabled collaborative tasks in distributed settings and across different devices or platforms. The research on these systems has predominantly focused on how to compensate for the lack of cues characterizing distributed settings.

Only recently has the research started exploring unprecedented opportunities that collaborative technologies offer with respect to face-to-face work conditions. A few recent studies have drawn on models from social psychology to develop tools that can help groups self-regulate their collaborative interactions. DiMicco and collaborators [10] have shown experimentally that shared visualizations that externalize aspects of the communication process can help collocated working groups to communicate more effectively. The presence of the visualization influences the level of participation of the members and the information sharing process during group decision-making. Studies of computer-supported communities have shown that member participation or community development can be influenced using appropriate tools (e.g., [2]).

A few studies on bias in groups have also show that collaborative technology can reduce specific types of bias in decision-making (e.g., [3]). Smallman et al. [16] and Billman et al. [4] have shown that appropriate visualization tools can reduce confirmation bias in intelligence analysis.

Overall, collaborative visualizations and collaborative tools can be used empower groups to process massive amount of data (information coverage and categorization) and provide information about the group process so that the group can become aware of and self-correct biases in the course of the decision-making activity. Situations become more complex in remote cooperation with mobile devices, which is often the case of emergency management work. Biases and limitations can increase because the type of representations and devices are diverse. 


\section{Addressing Sampling Bias and Limited Capacity}

Scenario: Three experts are planning an emergency management operation: they need to find the best solution available for evacuating a family from a flooded area to a shelter. The three experts, Public Works, Environmental, and Mass Care expert, collaborate through a geo-collaborative system. Each expert has both unique rolespecific information and shared information. For example, the Public Works expert is a civil engineer who has critical information on roads, bridges, and public infrastructures. For two of the four available shelters the three experts receive the same information regarding problems of common interest. For the other two shelters they have information regarding problems of role-specific interest. After a full round of sharing about the most prominent issues for each expert, they realize that they have very little time left, thus their group discussion gradually focuses on issues that they have in common. As a result, the information about first two shelters is discussed more exhaustively than the information about the second two shelters. The routes to the second pair of shelters are explored in less detail. Even though they had an equivalent amount of relevant information between the two pairs of shelters, several pieces of critical role-specific information is omitted or ignored in the discussion, which prevents them from finding the best solution. In the last part of the task, after sharing a large number of annotations, the collaborators feel overloaded. The map gets cluttered around the shelters, making it difficult for them to find what they need.

\section{Storyboarding}

Users filter out detailed information added to the map in two possible ways: by level of abstraction and by role. Users can switch among different levels of abstraction from 'Notes', which shows all the text content; 'Dots', which displays only role-based color-coded dots to present where annotations are; and 'Number,' which sums up the total number of annotations by shelter. In the experiment we observed that participants knew their own role-specific information and thus paid selective attention to the information added by their teammates. Users can toggle the display of role information to make the interface clearer (Figure 2, A).

The bar chart of annotation presents an intuitive visualization of the aggregation results. The bar chart (Figure 2, B) shows the information aggregated by shelters. We observed that people compared options one with another in the face to face experiment situation. By showing the annotation categorized by shelters, people may more easily perceive what are the shelters with the least constraints. Moreover, the annotation tool records the annotation and also the users' discussion activity about that. The annotation counter of a specific annotation increases as the users' click on it (Figure 3, A). 
By countering and showing the results that some the shelters are over-discussed with respect to others, the system could remind the users of this imbalance.

The timeline graph (Figure 2, C) shows the discussion activity of each expert as time goes by. The squares represent the activity of editing annotations (add and revisit). Different colored squares refer to different shelters. By tracking the process of individual analysis, we can see whether there are 'leaders' who talk a lot in the discussion process and 'followers' who keep silent in the most time, to build a model of the sharing process by the team (e.g., [10]).

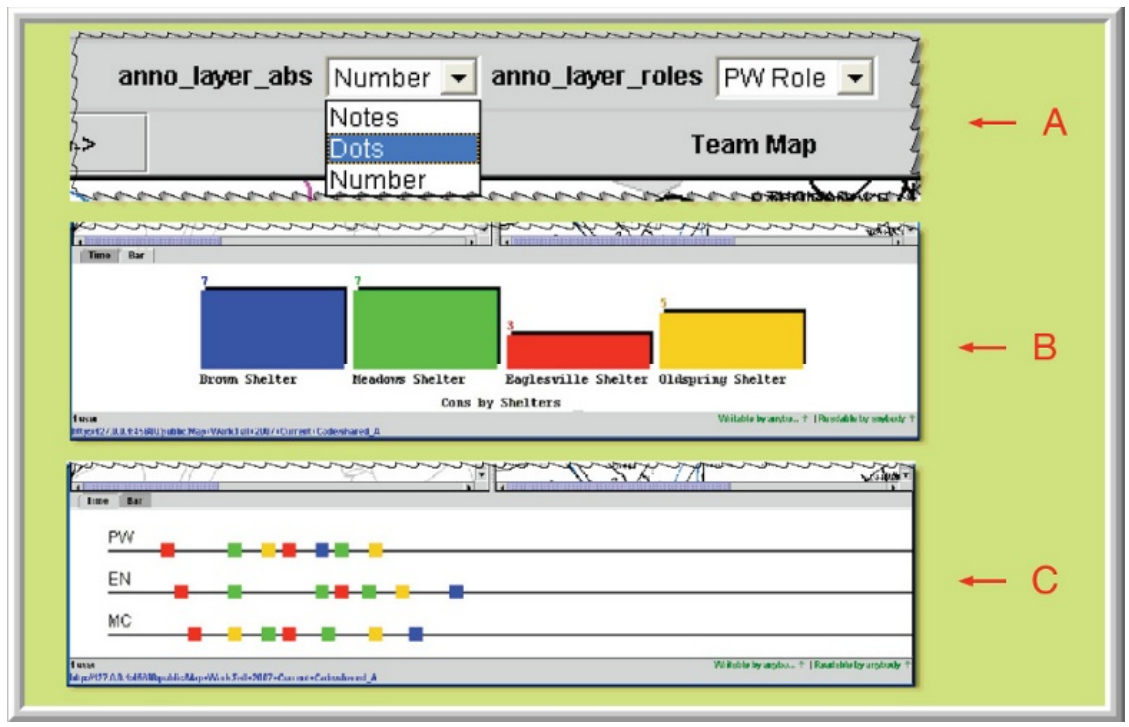

Figure 2. The augmented prototype. A: Filtering tools. B: Bar chart visualization by shelter. C: Timeline visualization by roles.

\section{Supporting Aggregation}

Scenario: A group of experts is training to plan potential emergencies on a shared map. The goal is to become increasingly efficient in sharing information and making optimal judgments as a well-coordinated team. When they solve the first emergency scenario the Public Works expert initiates the sharing process by suggesting his preferred shelter and summarizing the infrastructure problems affecting the other three shelters. The Mass Care and Environmental experts follow him by doing the same. All three roles are able to identify the annotations relevant to the shelters by tagging them appropriately. Then the Mass Care expert annotates the map with a "*non-spatial" 
note, where he suggests that they collect in 'summary' annotations non-spatial information on each shelter. This helps the team to quickly compare the four solutions. Thus, as they start the second scenario, the Environmental expert proposes that they start from summarizing non-spatial information shelter by shelter. He suggests: "Let's do the quick summaries as we did the last time", but he would have rather preferred to reuse directly the Mass Care's "*non-spatial" note from the last scenario. Also, he would have preferred to be able to quickly distinguish spatial from non-spatial concerns while they review the information.

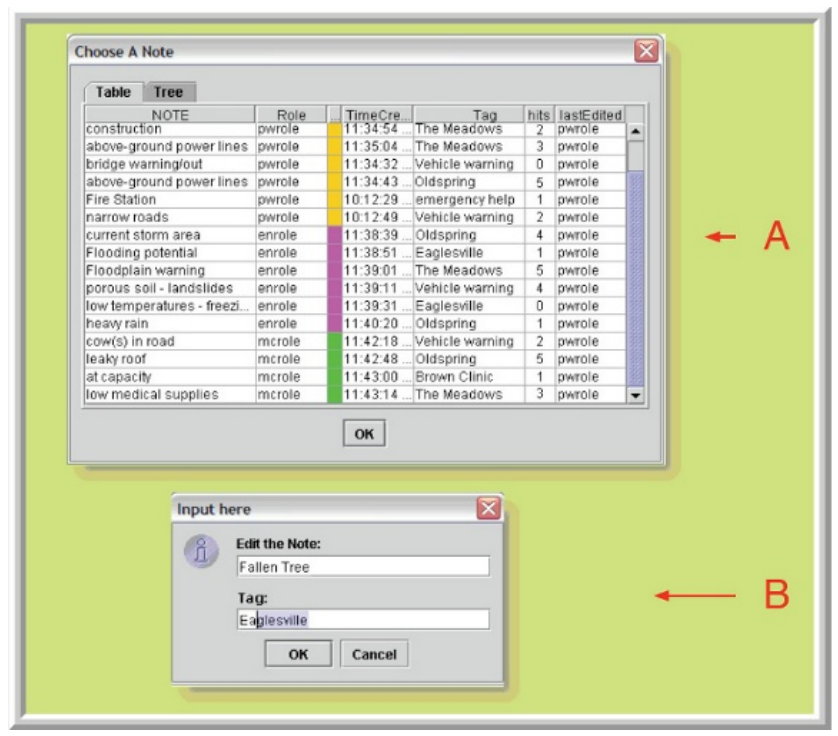

Figure 3. Annotation tools. A: annotation list showing contextual information and tags. B: Tag suggestion via an auto-complete when entering text.

\section{Storyboarding}

In addition to adding annotations to the maps, users define tags to classify and categorize these annotations. When editing an annotation, users not only can alter the label but also the tag field of the annotation as in Figure 3, B. By doing so, users can make use of tags, or shared categorizations, as they work together to group information and help coordinate their efforts. As the group works together, the system can give access to previously-used tags to let members leverage prior work, reuse shared conventions, and maintain consistent terminology. Figure 3, B shows a tag suggestion as a user edits the tag field. Observations from the experiment showed that teammates often asked each other which notes were relate to which shelters and, thus, which other 
notes. Furthermore, groups would add information not related to any specific locations represented on the map that they felt was valuable to the decision-making process.

The annotation tool provides a field for users to enter tags whenever they add an annotation to the map, though a tag is not required for a note to be added to the map. The annotation list tool - which provides information on the creator of the note, the last editor, and the time the note was created - has a field for the notes' tags, allowing for users to edit both the notes' text and their tags (Figure 3, A). As a result, users will be able to group the information that they have provided to one another on the map and better understand the contexts surrounding the notes and how they are interrelated. A benefit of these tags will be the ability for users to group their "nonspatial" information for easier reference regardless of its location on the map.

\section{Discussion}

An initial prototype for annotation tools has been developed. Currently, users can add annotations, sort them according to role. The visualization tools are in development. We are focusing on aggregating annotations, linking annotations and targets on map, and showing process timelines.

Upon the completion of tools to support group annotations and process visualization, we plan to extend our research into the two directions. First, we will study decision-making in a dynamic information space. Rapidly changing information may increase affect decision-making accuracy and judgment bias. Extending our previous study on complex decision-making in static information environments, we will examine team performance in dynamic environments and the benefits of annotations and process visualization tools through lab experiments. Second, we will develop a theoretical model on team behaviors in collaborative decision-making based on data from experiments. We aim to model team behaviors and common ground (CG) processes in complex decision-making (e.g., key factors and the critical stages of grounding in geocollaboration), and use this model to further inform the design of collaboration systems. This model can also guide our efforts to design software agents or recommendation systems to assist teams in complex decision-making [15; 10]. Previously, we have developed a preliminary empirical model on common ground processes $[5 ; 8]$.

Group annotations and process visualization will have a tangible impact on our research on CG. Our interests in knowledge sharing in collaboration go beyond shared mental representations, or shared mental model, per se, and concentrate on the process of constructing such representations, or CG process [14]. Originated from research on language [7]. CG is defined as "the things that we know about what is known by the person we are talking to"[14, p. 270]. We move beyond the scope of communication and model the CG process in the context of computer-supported cooperative work. 
Acknowledgements We thank the Office of Naval Research for the support (CKI program, award N000140510549). We thank Helena Mentis, Dejin Zhao, and our colleagues at Penn State.

\section{References}

1. André P., Wilson M. L., Russell A., Smith D. A., Owens A. (2007) Continuum: Designing Timelines for Hierarchies, Relationships and Scale, Proc. UIST 2007, Rhode Island.

2. Beenen, G., Ling, K., Wang, X., Chang, K., Frankowski, D., Resnick, P., et al. (2004). Using social psychology to motivate contributions to online communities. In Proceedings of CSCW. New York: ACM Press.

3. Benbasat, I. and Lim, J., 2000. Information technology support for debiasing group judgments: an empirical evaluation. Organ. Behavior \& Human Decision Processes, 83, 167-183.

4. Billman, D., Convertino, G., Pirolli, P., Massar, J.P., Shrager, J. (2006). Collaborative intelligence analysis with CACHE: bias reduction and information coverage. PARC, Tech Rep, UIR-2006-09.

5. Carroll J. M., Convertino G., Ganoe C., Rosson M.B. (to appear): Toward a Conceptual Model of Common Ground in Teamwork, In Letsky M. Warner N., Fiore S., \& Smith, C., Macrocognition in Teams. Amsterdam, Elsevier.

6. Carroll J.M., Mentis M., Convertino G., Rosson M.B., Ganoe C., Sinha H., Zhao D. (2007) Prototyping Collaborative Geospatial Emergency Planning. Proceedings of ISCRAM 2007.

7. Clark H. H. (1996). Using Language. Cambridge, UK: Cambridge University Press.

8. Convertino G., Mentis H., Rosson M.B., Carroll J.M., Slavkovic, A. (to appear). Articulating Common Ground in Cooperative Work: Content and Process. In Proceedings of CHI 2008 Conference. April 5-10, 2008, Florence, Italy.

9. Convertino G., Zhao D., Ganoe C., Carroll J.M., Rosson M.B.: (2007). A Role-based Multi-View Approach to support GeoCollaboration, Proceedings of HCI International 2007, Beijing, China.

10. DiMicco, J.M., Hollenback, K.J., Pandolfo, A., and Bender, W., (2007). The Impact of Increased Awareness while Face-to-Face. Human-Computer Interaction, 22, 47-96.

11. Ganoe C.H., Somervell J.P., Neale D.C., Isenhour, P.L.,Carroll, J. M., Rosson, M.B., McCrickard, D. S. (2003). Classroom BRIDGE: using collaborative public and desktop timelines to support activity awareness, In Proc. of UIST 2003, 21-30, Vancouver, Canada.

12. Kahneman, D. Slovic P. and Tversky A. (1982). Judgement under uncertainty: Heuristics and biases. New York: Cambridge University Press. 201-208.

13. McCarthy, J.C., Miles, V.C., Monk, (1991). An experimental study of common ground in textbased communication, In Proceedings of CHI 1991, ACM Press, 209-215.

14. Monk, A. (2003). Common Ground in electronically mediated communication: Clark's theory of language use, in J.M. Carroll, Towards a multidisciplinary science of HCI. MIT Press.

15. Nan N., Johnston E. W., Olson J. S., \& Bos N. (2005). Beyond Being in the Lab: Using MultiAgent Modeling to Isolate Competing Hypotheses, Proc. CHI 2005. ACM. 1693-1696.

16. Smallman H. S. (in press): JIGSAW - Joint Intelligence Graphical Situation Awareness Web for Collaborative Intelligence Analysis. In Letsky M. Warner N., Fiore S., \& Smith, C., Macrocognition in Teams. Amsterdam, Elsevier.

17. Stasser, G \& Titus, W. (2003). Hidden profiles: A brief history.Psychological Inquiry,3-4, 302-11.

18. Stout, R., Cannon-Bowers, J.A., Salas, E. (1996). The role of shared mental models in developing team situation awareness: Implications for training. Training Research J.., 2, 85-116.

19. Weng, C., and Gennari, J.H. (2004). Asynchronous collaborative writing through annotations, Proceedings of CSCW '04, Chicago, ACM Press, 578-581. 